\title{
Substantiation of rational parameters of the root crops separator with a rotating inner separation surface
}

\author{
Rim Khamaletdinov, Vladimir Martynov, Salavat Mudarisov, Ildar Gabitov, Eduard Khasanov, \\ Anton Pervushin
}

Federal State Budgetary Educational Establishment of Higher Education, Bashkir State Agrarian University, Ufa, Russian Federation

\begin{abstract}
The aim of the paper is to justify the design and rational parameters of the secondary post-treatment separator of potato heap in the form of a truncated cone with a rotating internal separating surface which ensures the separation of potato tubers from soil lumps commensurate with them. Based on the analysis of works devoted to the process of potato heap separation, including on inclined surfaces, as well as the analytical calculations, it was concluded that it is possible to create a device for separating potato tubers from soil lumps in the form of a rotating truncated cone with an internal separating surface. As a result of the research, the design of the secondary post-treatment separator of potato heap in the form of a rotating drum in the form of a truncated cone with a separating roller. Laboratory and field tests have shown that with appropriate adjustments, separation of up to $80 \%$ of soil impurities commensurate in size with potato tubers is provided.
\end{abstract}

\section{Introduction}

The plans for the implementation of food security most countries have defined the nomenclature of strategically important crops, among which the potato always occupies one of the leading positions (Devaux and Ortiz, 2014; FAO, 2018). A need for significant financial investments, significant labor costs in the cultivation and harvesting especially on heavy wetlands limit the produc-

Correspondence: Rim Khamaletdinov, Federal State Budgetary Educational Establishment of Higher Education, Bashkir State Agrarian University, Ufa, Russian Federation.

E-mail: rkamaletdinov87@yahoo.com

Key words: Potato harvester; potato tubers; soil lumps; rotating truncated cone; separation; vanishing angles; virtual modelling.

Received for publication: 4 July 2019.

Accepted for publication: 10 October 2019.

(C) Copyright: the Author(s), 2019

Licensee PAGEPress, Italy

Journal of Agricultural Engineering 2020; LI:997

doi:10.4081/jae.2019.997

This article is distributed under the terms of the Creative Commons Attribution Noncommercial License (by-nc 4.0) which permits any noncommercial use, distribution, and reproduction in any medium, provided the original author(s) and source are credited. tion of this crop in a number of soil and climatic zones (Abhilash et al., 2016). At the same time, the use of even the most advanced technologies and methods of storage does not guarantee the safety of the crop if the quality of the stored products does not meet agricultural requirements (Ebrahem et al., 2011). Among the agrotechnical indicators, the most important are the presence of soil impurities and damaged tubers in the similar heap which inevitably leads to the loss of a significant part of the crop if the agrobiological background is unfavorable (Agu et al., 2015; Khamaletdinov et al., 2018). This state of affairs is largely due to the fact that in modern potato harvesters the main way to separate potato tubers from impurities is the separation of potato heap on bar elevators in thickness after the preliminary destruction of soil lumps to pass sizes (Bishop and Monder, 1983; Gao et al., 2010; Klindtworth and Sonnen, 2014; Fomin, 2018). In this case, the magnitude of the external forces applied to the tuber formation is limited by the condition of damage to the tubers. However, on heavy and waterlogged soils, the impact of forces in the range of allowable values is insufficient to destroy the bulk of lumps, which leads to an increased soil content, and with an increase in the impact forces leads to injury to tubers (Mathew and Hyde, 1997; Mollah et al., 2018). Despite numerous works devoted to the development of separating bodies, the principle of work is the use of the difference in the characteristics of potato tubers and soil particles, such as hardness, specific gravity, elasticity, friction coefficients of sliding and rolling, aerodynamic properties, the ability to absorb and reflect rays and others, the task of creating devices for separating potato tubers from soil lumps commensurate with potato tubers remains unresolved (Klindtworth and Sonnen, 2014; Agu et al., 2015; Khasanov et al., 2018).

\section{Materials and methods}

\section{Research design}

The analysis of physical and mechanical characteristics of potato heap components shows that the greatest difference of separation characteristics is observed in the rolling angles of tubers and soil lumps (Zangeneh et al., 2015; Jamróz et al., 2018).

Figure 1 shows the density distribution of the angles of rolling the tubers of potato varieties Nevsky and lumps of soil is loam type indicating the presence of minor areas of overlap of this symptom. It is no coincidence that currently the most promising for the separation of commensurate in size potato tubers and soil particles are devices that operate on the principle of an inclined finger slide. At the same time the proposed constructive solutions based on the division on this basis have a number of drawbacks.

First, the components of the heap move when separating 
towards each other (in the counterflow), and secondly, the adjustment parameters (angles, kinematic modes of operation, elasticity of the fingers, etc.) are determined based on the average physical and mechanical characteristics of the heap components, which does not allow these devices to ensure complete separation of root crops from soil lumps. Thus, the authors propose the design of the separator in the form of a flexible moving infinite tape in the form of a body of rotation, for example, a truncated cone, the design scheme of which is shown in the Figure 2.

\section{Mathematical model}

Taking into account the shape and kinematics of the interacting surfaces, let's consider the moving components of the heap in a cylindrical coordinate system $\rho, \varphi, z$. Polar radius $\rho$ determines the distance to the centre of mass of the components from the rotation axis of the cone $O z$. The point of contact of the interacting surfaces is on the ray passing perpendicularly through the axis $O z$ and the centre of component mass.

The deviation of the radius $\rho$ from the vertical is determined by the polar angle $\varphi$. The movement along the axis of rotation of the

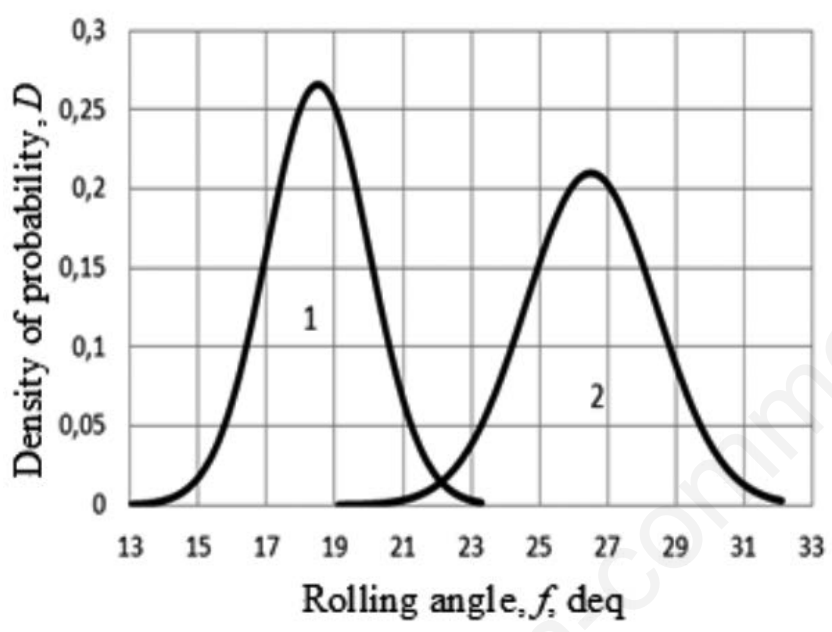

Figure 1. Density distribution of angles rolling: 1) potato tubers; 2) soil lumps. cone is estimated by the coordinate $z$. The radial acceleration of the center of mass of the component is determined by $\ddot{\rho}-\rho \varphi^{2}$, trasversal acceleration $-\rho \ddot{\varphi}+2 \rho \varphi$, respectively the velocities and accelerations along the axis $O z$ are equal to $\dot{z}=\dot{\rho} / \operatorname{tg} \alpha$ and $\ddot{z}=\ddot{\rho} / \operatorname{tg} a$. The component with the mass $\mathrm{m}$ is affected by the following forces: gravity $G=m g$, the normal reaction $N$ and the friction force $f N$, where $f$ is the coefficient of friction of the component on the cone surface. After projecting all the acting forces on the cylindrical coordinate axes, taking into account the inertial component of the point mass, the system of differential equations of component motion is as follows:

$$
\left.\begin{array}{l}
m\left(\ddot{\rho}-\dot{\rho}^{2}\right)=G \sin \beta \cos -N \cos \alpha-f N \dot{\rho} / \sqrt{v_{o}^{2}+\dot{\rho}^{2} / \sin ^{2} \alpha} ; \\
m(\rho \ddot{\rho}+2 \dot{\rho})=-G \sin \beta \sin -f N v_{o} / \sqrt{v_{o}^{2}+\dot{\rho}^{2} / \sin ^{2} \alpha} \\
m \ddot{z}=m \ddot{\rho} / \operatorname{tg} \alpha=N \sin \alpha-G \cos \beta-f N \dot{\rho} /\left(\operatorname{tg} \beta \sqrt{v_{o}^{2}+\dot{\rho}^{2} / \sin ^{2} \alpha}\right)
\end{array}\right\}
$$

where $\sqrt{v_{o}^{2}+\dot{\rho}^{2} / \sin ^{2} \alpha}$ is the absolute velocity component $V_{\kappa}$; $v_{o}$ is the relative circumferential velocity of the component equal to:

$$
v_{o}=\rho_{1}\left(\dot{\varphi}-\omega_{0}\right)
$$

where $\omega_{0}$ is the angular velocity of rotation of the conical drum; $\rho_{1}$ is the polar radius of the contact point of the component with the inner surface of the truncated cone which, taking into account the radius $\rho_{k}$ of the component, is defined as the sum of:

$\rho_{1}=\rho+\rho_{k} \cos a$,

To exclude the unknown normal reaction $N$ from the system of equations, the first equation from the third equation of the system can be subtracted, previously dividing it into tga. After performing the transformation, the following formula can be obtained:

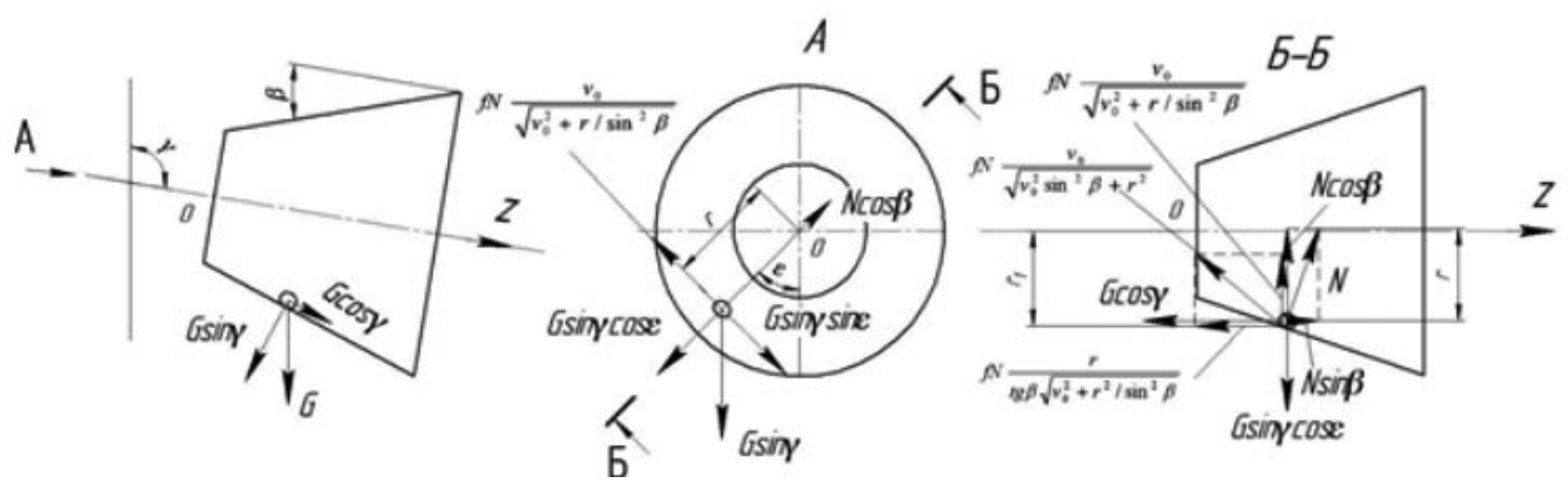

Figure 2. The scheme of forces acting on the moving component of the potato heap on the inner surface of the rotating truncated cone of the separator. 
After replacing the normal reaction with (4), the system of equations of motion of the component in the rotating truncated cone takes the form

$$
\left.\begin{array}{l}
\ddot{\varphi}=-\frac{1}{r}\left\{2 \dot{\rho} \dot{\varphi}+g \sin \beta \sin \varphi+\left[\rho \dot{\varphi}^{2}+g(\cos \beta \cdot \operatorname{tg} \alpha+\sin \beta \cos \varphi)\right] \frac{f v_{o} \sin \alpha \cos \alpha}{\sqrt{v_{o}^{2} \sin ^{2} \alpha+\dot{\rho}^{2}}}\right\} \\
\ddot{\rho}=\sin \alpha\left[\rho \dot{\varphi}^{2}=g(\cos \beta \cdot \operatorname{tg} \alpha+\sin \beta \cos \varphi)\left(\sin \alpha-\frac{f \cdot \dot{\rho} \cos \alpha}{\sqrt{v_{o}^{2} \sin ^{2} \alpha+\dot{\rho}^{2}}}\right)-g \cos \beta \cdot \operatorname{tg} \alpha .\right.
\end{array}\right\}
$$

The numerical solution of the system (5) in the simulation program of imitation model allowed determining the current values of the angle $\varphi$ and radius $\rho$ for any time $t$ or the angle of rotation of the cone $\psi=\omega_{0} t$. The initial radius $\rho=\rho_{0}$, respectively, is equal to $\rho_{0}=R_{\min }-\rho_{K} \cos \alpha$, where $R_{\min }$ is the radius of the smaller base of the truncated cone. This radius at the given values of the angle $\alpha$, the radius of the greater base $R_{\max }$ and the height $h_{z}$ of the truncated cone is:

$R_{\min }=R_{\max }-h_{z} \cdot \operatorname{tg} a$

The current coordinate $z$ for a known radius $\rho$ is

$z=\left(\rho+\rho_{k} / \cos a-R_{\min }\right) / \operatorname{tg} a$

Figure 3A shows the lateral displacement of the heap component when moving along the inner surface of a cone rotating at an angular speed $\omega=6 \mathrm{c}^{-1}$, with a length of 1 meter, an angle $\alpha=30^{\circ}$ and a diameter of a smaller base $R_{\min }=0.6 \mathrm{M}$ with different friction coefficients. In Figure 3B the same is given with the coefficient of friction $f=0.7$ at different angular velocities of rotation of the drum.

Lateral displacement of the trajectory of the heap components when moving along the inner surface of a truncated cone with an angle forming $\alpha=30^{\circ}$ :

a) at friction coefficients: $1-f=0.3 ; 2-f=0.5 ; 3-f=0.7 ; 4-\mathrm{f}=0.9$;

b) angular velocities: $1-\omega=3 \mathrm{c}^{-1} ; 2-\omega=6 \mathrm{c}^{-1} ; 3-\omega=9 \mathrm{c}^{-1} ; 4-$ $\omega=12 \mathrm{c}^{-1}$

\section{Calculations and experiments}

The limit angles of lifting of these bodies (Figure 4) and the modes of motion stabilization, i.e. rolling without slippage, were determined, since the latter leads to peeling of the tubers skin. Thus, it follows from the data obtained that the action of centrifugal forces provides additional divergence of rolling angles of potato tubers and soil lumps. For single tubers and soil lumps on a flat surface, the difference in rolling angles is on average 110 and has overlap zones. In a rotating drum, this parameter can reach 36-400. It was found that the transition from static to kinetic mode, and thus the change in the values of the friction coefficients requires a certain time, i.e. there is a transition phase the effect of which on the trajectory of the separated components has not been considered. Description of the process of rolling different bodies of rotation on inclined surfaces is one of the fundamental problems of theoretical mechanics. Many other scientists were engaged in the development of the bases of this problem solution. However, despite this fact analytical descriptions were obtained only for particular simplified cases. Particular solutions close to real ones can be obtained only by computer implementations of numerical models. Based on the analysis of the proposed methods of solving such problems it is believed that the most effective one is the use
A

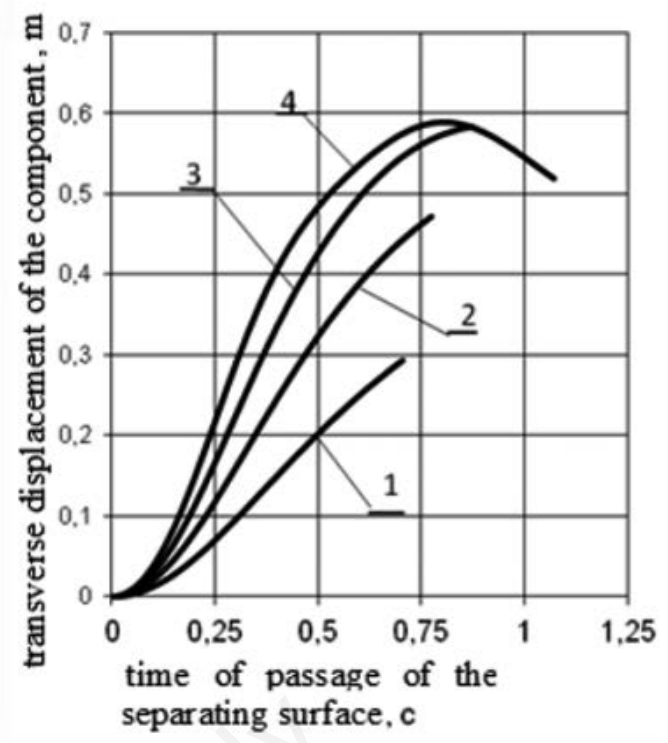

B

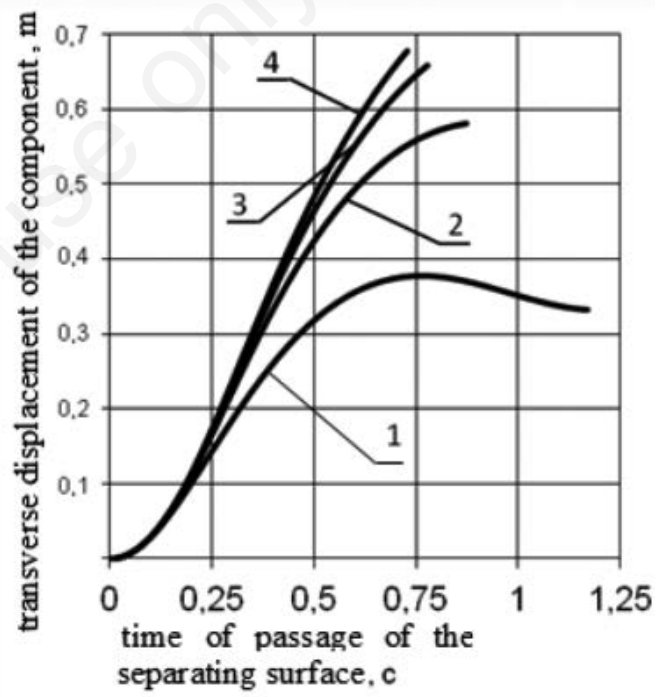

Figure 3. The lateral displacement of the heap component when moving along the inner surface of a cone.

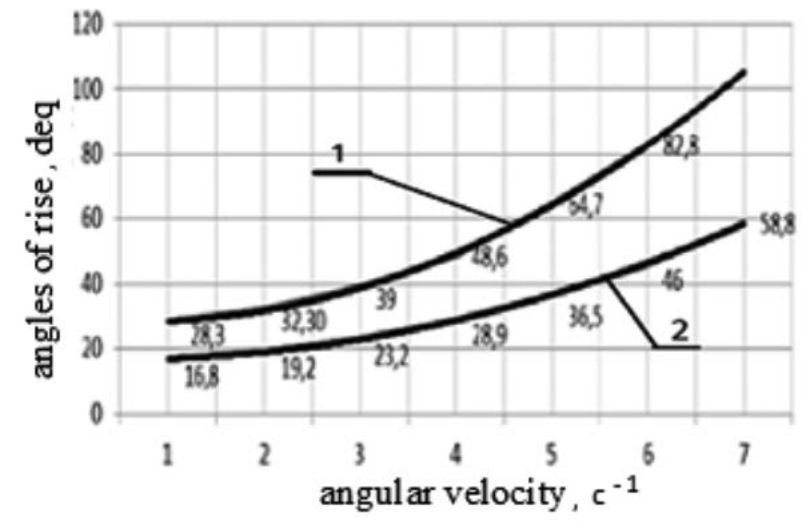

Figure 4. Limit angles of rise: 1) lumps of soil; 2) potato tubers depending on he angular velocity $\omega$ atr $=0.45 \mathrm{~m}$. 
of virtual modelling environment for this case (Bishop and Monder, 1983).

\section{Results and discussion}

As a result of the carried out machine experiments with the generation of friction properties of the components of the separated heap according to the normal law and at different frequencies of rotation of the drum, it was revealed that the trajectory of the components of the heap has a wave nature (Figure 5).

With that, the change in the speed of the drum on the trajectory of the potato affects slightly, in contrast to the soil lumps which oscillate, rising and rolls with a higher frequency and amplitude. If the components are removed from the separating surface in the first quarter of the oscillation, the intersection of trajectories can be avoided. The difference in the elevation angles of soil lumps and tubers can be used as a dividing sign and the possibility of separating tubers from soil lumps in unidirectional flows can be considered, which is consistent with the results of Sibirev et al. (2019). The analysis allowed drawing a conclusion about the need to find constructive-kinematic parameters at which the trajectories of the moving components of the heap do not overlap and the difference of deviations on the $\mathrm{X}$-axis at the descent was minimal.
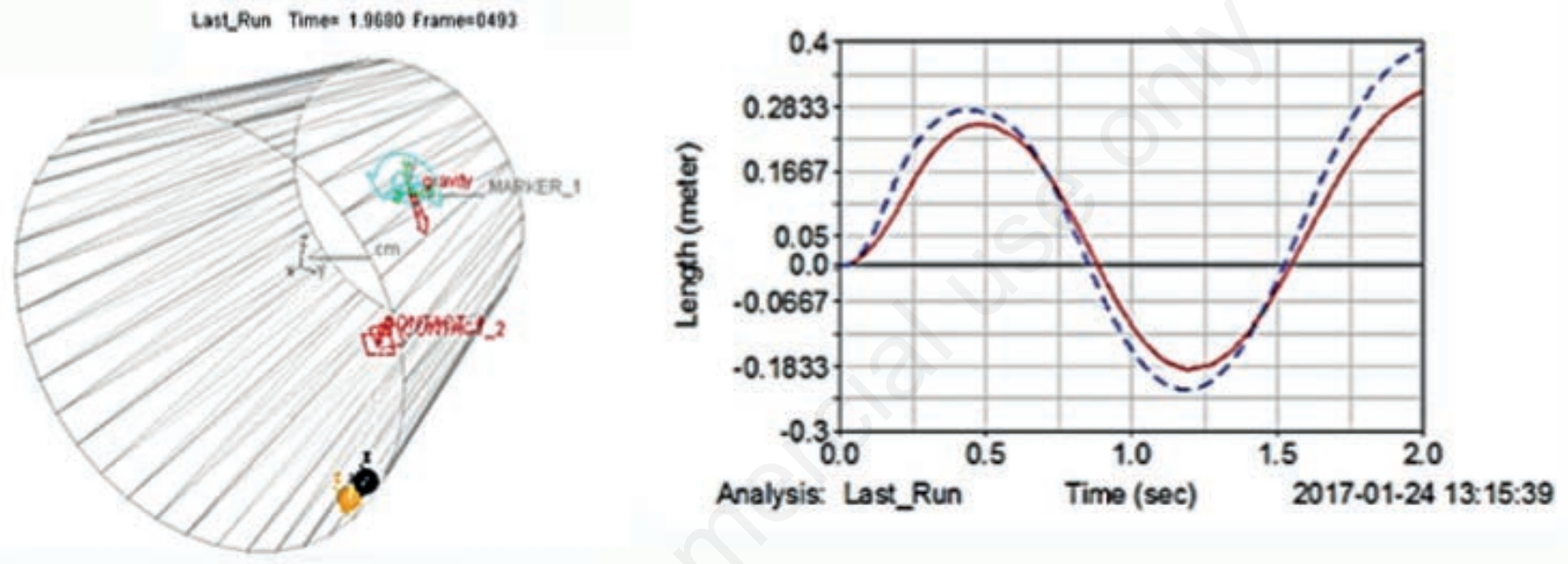

Figure 5. The fragment of analysis of trajectories of movement of ideal soil lumps and potato tubers in the horizontal plane when moving along the inner surface of a rotating rubber truncated cone at: $\omega^{\prime}=12,56 \mathrm{c}^{-1}, \alpha=15^{\circ}$ and $\beta=85^{\circ}$.
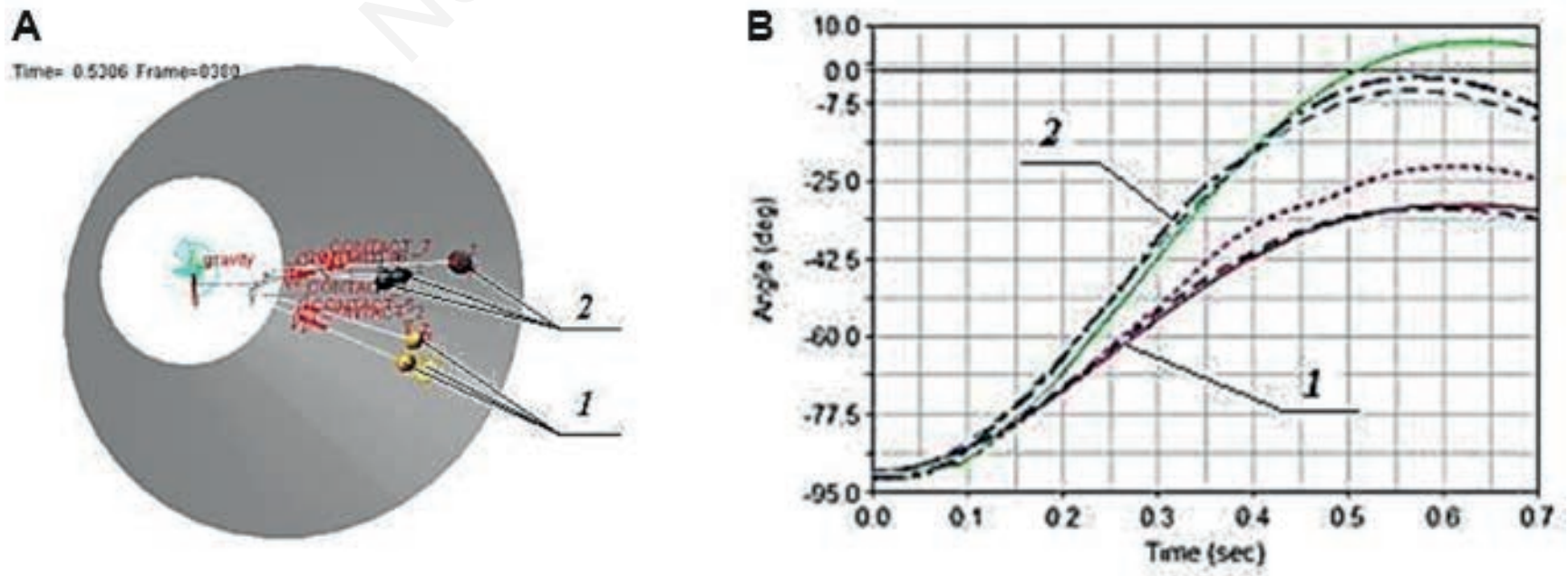

Figure 6. A fragment of the analysis of the trajectory (A) and graphs of the angles of raise (B) on the inner surface of the conical separator of different shapes: 1) potato tubers; 2) soil lumps. 

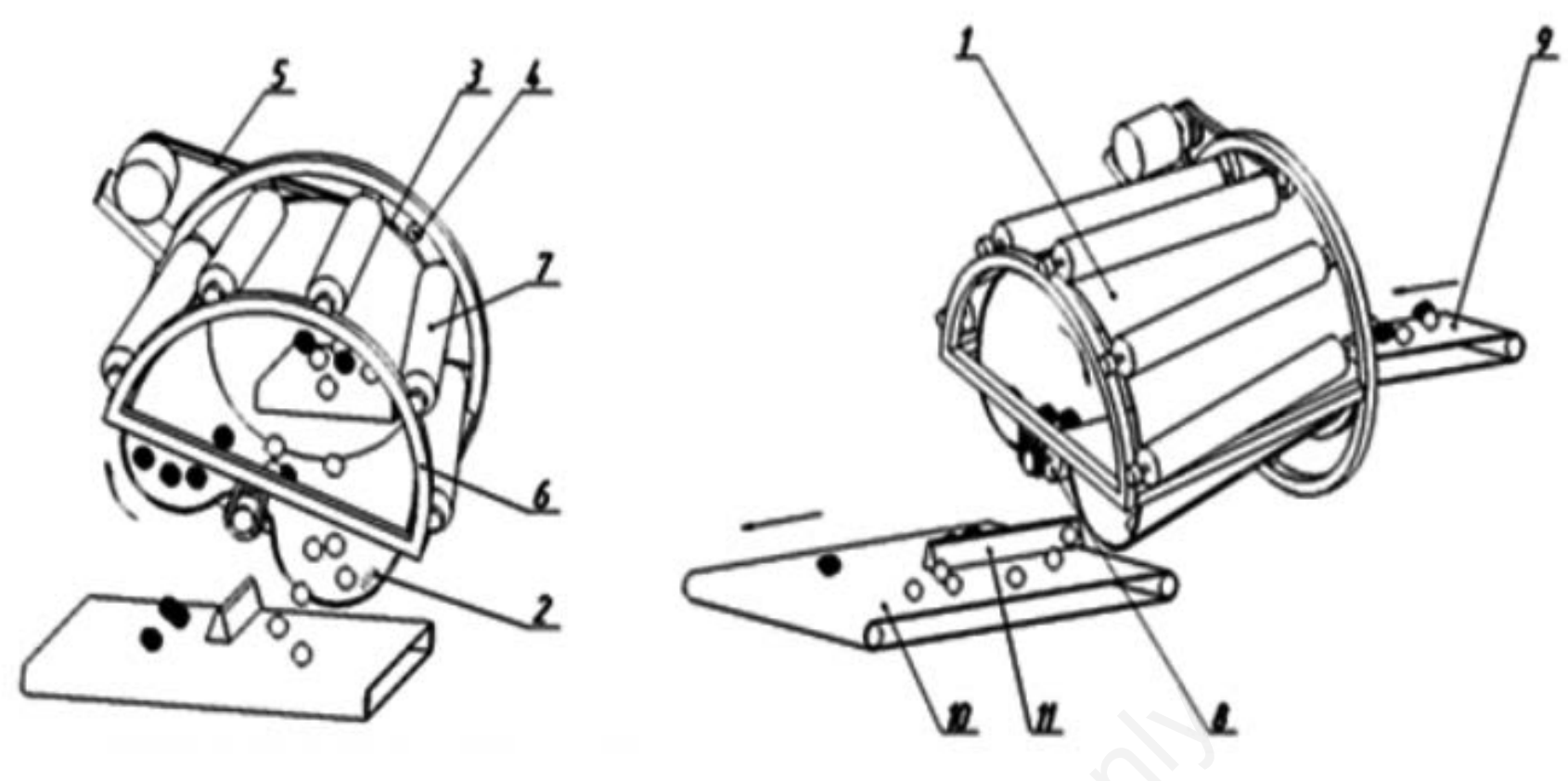

Figure 7. Drum separator of potato heap in the form of truncated cone (numbering in the text).

Figure 6 shows a fragment of the analysis of the trajectory of potato tubers and soil lumps in the form of an ideal ball, ellipse and pancake in the virtual model of a conical drum with an angle of $\alpha=$ $30^{\circ}$, illustrating the possibility of obtaining a significant difference between the points of descent of potato tubers and soil lumps. In the considered virtual model, the maximum difference between the angles of descent at $\omega=12,5 \mathrm{c}^{-1}$ was achieved almost at the time of the descent of potato tubers and soil lumps from the separating surface. To obtain the maximum difference, it is sufficient to increase slightly the angle of inclination of the rotation axis $\beta$.

The results obtained are fully consistent with the results of other scholars who studied similar processes in the harvesting of fodder beet (Mathew and Hyde, 1997; Gao et al., 2010; Chakwizira et al., 2018; Malcolm et al., 2019).

The validity of the results obtained on virtual models was tested on the experimental setup, the scheme of which is shown in Figure 7 . The proposed device contains a separating surface made in the form of a flexible infinite tape in the form of a body of rotation, for example, a truncated cone drum 1, the inner side of which is provided with elastic fingers 2 . The smaller base of the cone drum 1 is fixed to the cylindrical rim 3 rotating on the guide rollers 4 by means of the drive 5 . Along the side surface of the drum 1 on the frame 6, support rollers 7 are installed. In the lower part of the frame 6 , a deforming roller 8 is placed capable of moving in longitudinal and transverse directions and changing the angle of inclination, and also providing the creation on the inner side of the separating surface in the course of rotation of the convexity of variable height increasing to the lower base of the drum 1. Frame 6 is made with the ability to change the angle of inclination relative to the horizon. Supply of the heap is carried out by the conveyorfeeder 9 .

\section{Conclusions}

The proposed friction separator in the form of a truncated cone separator is a new patent-protected promising class of devices for the separation of root crops from the soil. It provides the possibility of separating similar in size characteristics of soil lumps from potato tubers in a unidirectional flow through a complex of physical and mechanical parameters. The use of software systems of virtual modelling, in particular MSC ADAMS, in combination with simulation, allows you to develop and select parameters in the online mode with a significant reduction in the time to find new solutions and optimize the design and technological parameters of the designed devices. Based on the yield of $250 \mathrm{~kg} / \mathrm{ha}$ and the performance of a single-row harvester $0.25 \ldots 0.35 \mathrm{ha} / \mathrm{h}$ the inlet diameter of the drum should be at least $0.6 \mathrm{~m}$, the length of the side surface is 1.2 $\mathrm{m}$ and the angle of inclination to the horizon is $28 . . .35$, the drum speed has a smooth control range from 6 to $10 \mathrm{c}^{-1}$. Installation of the proposed separator on the potato harvester will provide at least a two-fold reduction in the content of soil particles in the pile.

\section{References}

Abhilash PC, Tripathi V, Edrisi SA, Dubey RK, Bakshi M, Dubey PK, Ebbs SD. 2016. Sustainability of crop production from polluted lands. Energ. Ecol. Environ. 1:54-65.

Agu KC, Nweke GU, Awah, HNS, Okeke BC, Mgbemena ICC, Okigbo RN, Ngenegbo UC. 2015. Fungi Associated with the Post-Harvest Loss of Sweet Potato. Int. J. Res. Stud. Biosci. 3:32-7. 
Bishop KF, Monder UF. 1983. Mechanization of potato production and storage. Publishing House Kolos, Moscow, Russia, pp 256.

Chakwizira E, Teixeira E, Meenken E, Michel AJ, Maley S. 2018. Radiation use efficiency and biomass partitioning to storage roots in fodder beet crops. Eur. J. Agron. 92:63-71.

Devaux PR, Ortiz O. 2014. Potato for sustainable global food security. Potato Res. 57:185-99.

Ebrahem IZ, Ayman AE, Guidetti R. 2011. A new small potato planter for Egyptian agriculture. J. Agric. Engine. 42:7-14.

FAO (Food and Agricultural Organization of the United Nations). 2018. FAOSTAT. Available from: http://www.fao.org/ faostat/ en/Accessed: 29.06.2018.

Fomin A. 2018. Import substitution in the agro-industrial complex of Russia. Int. Agric. J. 61:1-14.

Gao G, Zhang D, Liu J. 2010. Design of a new soil-tuber separation device on potato harvesters. In International Conference on Computer and Computing Technologies in Agriculture. Springer, Berlin, Heidelberg, Germany, pp 604-612.

Jamróz E, Juszczak L, Kucharek M. 2018. Investigation of the physical properties, antioxidant and antimicrobial activity of ternary potato starch-furcellaran-gelatin films incorporated with lavender essential oil. Int. J. Biol. Macromol. 114:1094-101.

Khamaletdinov RR, Gabitov II, Mudarisov SG, Khasanov ER, Negovora AV, Martynov VM, Stupin VA, Gallyamov FN, Farkhutdinov IM, Shirokov DY. 2018. Improvement in engineering design of machines for biological crop treatment with microbial products. J. Engine. Appl. Sci. 13:6500-4.

Khasanov ER, Negovora AV, Martynov VM, Stupin VA, Gallyamov FN, Farkhutdinov IM, Shirokov DY. 2018. Improvement in engineering design of machines for biological crop treatment with microbial products. J. Engine. Appl. Sci. 13: $6500-4$.

Klindtworth M, Sonnen J. 2014. Kartoffeltechnik. Braunschweig: Institut für mobile Maschinen und Nutzfahrzeuge, pp 164-171.

Malcolm BJ, Cameron KC, Curtin D, Di HJ, Beare MH, Johnstone PR, Edwards GR. 2019. Organic matter amendments to soil can reduce nitrate leaching losses from livestock urine under simulated fodder beet grazing. Agric. Ecosyst. Environ. 272:10-8.

Mathew RG, Hyde M. 1997. Potato impact damage thresholds. Trans. ASAE 40:705-9.

Mollah MAH, Hawlader AJ, Ali MR, Khatun R, Ahmed S, Mahmud MS, Hasan MA. 2018. Assessment of Technological Knowledge on Pre-and Post-harvest Agricultural Management System and its Economic Impacts in Bangladesh. Univ. J. Agric. Res. 6:79-90.

Sibirev A, Aksenov A, Dorokhov A, Ponomarev A. 2019. Comparative study of force action of harvester work tools on potato tubers. Res. Agric. Engine. 65: 85-90.

Zangeneh M, Omid M, Akram A. 2015. Integrated assessment and modeling of agricultural mechanization in potato production of Iran by Artificial Neural Networks. Agric. Res. 4:283-302. 